176 SUBSTANCE MISUSE IN PALLIATIVE CARE: AN AUDIT
EXPLORING REGIONAL PRACTICE IN IDENTIFYING
PATIENTS WITH SUBSTANCE MISUSE ACROSS
COMMUNITY, HOSPICE AND HOSPITAL SETTINGS

Penny Shepherd, Joanna Roberts, Kamilla Krueger, Sabine Auth, Elaine Mannering, Malcolm Cooper, Laura Chapman, Jenny Smith, Anthony Thompson. Woodlands Hospice, Willowbrook Hospice, Merseycare, Clatterbridge Cancer Centre, Marie Curie Liverpool, Countess of Chester Hospital, St Helens and Knowsley Teaching Hospitals

\subsection{6/spcare-2020-PCC.196}

Background Substance misuse (including alcohol) is a growing issue nationally with physical, psychological, social and spiritual consequences making it very relevant in palliative care, especially as patients often require medications for symptom control with the potential for misuse. Research and national guidance specific to palliative care is limited. It has been suggested substance misuse is under-recognised within palliative care and that patients should be routinely assessed for it.

Aims To evaluate regional practice and confidence among palliative care professionals in identifying patients with substance misuse as part of updating the 2009 regional guidelines on this topic.

Methods A retrospective multi-centre audit of palliative care professionals working across community, hospice and hospital settings in Mersey was conducted including questions on previous experience, screening and confidence in identifying patients with substance misuse.

Results 72 palliative care professionals responded (23\% community, $37 \%$ hospital, $40 \%$ hospice). A wide range of substance misuse had been encountered including alcohol (96\%), cannabis $(80 \%)$, intravenous heroin $(67 \%)$ and opioids $(65 \%)$.

Routine screening for substance misuse was uncommon: $21 \%$ for alcohol and $6 \%$ for other substance misuse. Only $6 \%$ routinely used a formal screening tool.

$63 \%$ of respondents felt fairly to very confident in their ability to identify substance misuse, $13 \%$ felt fairly to very unconfident. There was a high volume of responses regarding the need for general education and training relating to managing substance misuse.

Conclusions Within Mersey palliative care professionals have experienced patients presenting with a variety of types of substance misuse. Although many respondents felt confident in their ability to detect substance misuse, there was a lack of routine screening and formal screening tools were used infrequently. This could lead to under-recognition and a lack of appropriate support for patients. Clear education needs were identified and updated regional guidance is in the process of being created.

\section{EVALUATION OF THE EFFECTS OF FRAGONIA ESSENTIAL OIL ON SLEEP PATTERNS AND INSOMNIA}

Suzi Chester, Lyn Crecy, Amanda Wilkins, Kate Shorthose. St Margaret's Hospice, Somerset

\subsection{6/spcare-2020-PCC.197}

Background The Complementary therapy team had observed a pattern of patients commenting on improved sleep after receiving treatments with Fragonia essential oil. A literature search revealed that there is little research in this area to guide use of specific oils. The team decided to carry out an evaluation of use of the oil on a sample of patients to seek confirmation of a pattern which then might justify further investigation.

Method Sample of 20 patients from the day centre at St Margaret's Hospice, Somerset

Patients were asked on a scale of 1-10 to score their sleep pattern, from poor/no sleep to a restful/full nights sleep. Patients were given $25 \mathrm{ml}$ pots of base cream plus 5 drops fragonia, and asked to apply on the wrists before bed and then to document any changes in sleep patterns and duration of sleep. They were asked to apply the cream for a period of 7 days after which they were asked again to measure their sleep pattern on a scale of 1-10. They were also asked if they thought any other symptom had improved.

Results The results show that sleep was reported as improved among $85 \%$ patients. Other improvements were seen in tension/anxiety, pain and breathing. There was an average improvement of 3 points in the sleep scores for the 20 patients.

Comments included:

'better/longer sleep. Feel quite/more awake in the morning'

'the smell is very relaxing, slept well'

'very deep sleep until 9am from about midnight'

Conclusion This evaluation of the use of fragonia essential oil by patients in this way, appears to show a trend in improved sleep pattern. On the basis of this, the aim would be to carry out a piece of research, using validated scales and comparing cream with fragonia oil with a sample of patients with the cream alone.

\section{ENHANCED SUPPORTIVE CARE - MAKING A DIFFERENCE}

Kate Tredgett. Gloucestershire Hospitals NHS Foundation Trust

\subsection{6/spcare-2020-PCC.198}

Introduction Patients are often referred to palliative care at a stage when they are rapidly deteriorating and prognosis is short, meaning they receive a reactive service and have limited opportunity to benefit. The term 'palliative care' and its association with death for both professionals and patients, deters earlier referral to and engagement with the service. Palliative care can improve symptom control, quality of life and ensure patients' priorities and preferences are met. It can impact on interactions with other health care services.

Methods We developed a new service for all patients diagnosed with incurable upper gastro-intestinal cancers, delivering a proactive palliative care style of patient centred care earlier. This was delivered as the Enhanced Supportive Care (ESC) CQUIN. The ESC service was outpatient based and consultant led. Patients were identified through MDTs and rapidly offered assessment. Follow-up depended on needs. The IPOS questionnaire supported needs assessment. Patients were referred to other services as needed. Information was collected and comparison of outcomes made to data for a comparable period the preceding year.

Results Patients offered ESC or palliative care review at diagnosis increased (43\%-93\%)

The service was acceptable to most (85\% accepted)

Symptom burden was high - $82 \%$ had at least one severe/ overwhelming symptom at diagnosis

On follow-up, average severity score of 11/13 symptoms had improved 
Average length of hospital stay in the last 30 days of life fell, equating to a saving of 220 bed days or $£ 88000 /$ year

Patient/relative feedback was very positive.

Discussion The data suggests ESC is acceptable to and benefits patients. Fixed term funding is enabling the service to be expanded short term. Robust data collection and service analysis continue.

\section{A HOSPICE BASED RENAL SUPPORTIVE CARE SERVICE: AN EVALUATION OF THE FIRST 3 YEARS}

Jane Whitehurst, Sue Goodall, Ellie Hayter, Kevin Blackett. St Barnabas House

\subsection{6/spcare-2020-PCC.199}

Background St Barnabas House launched a Renal Supportive Care (RSC) Service in April 2016. This consists of a RSC Clinical Nurse Specialist (CNS), consultant support and joint working with the local secondary care renal team. Since 2016, 110 patients with advance renal disease (CKD 5) have been referred to our RSC service, approximately $3 \%$ of the hospice's total referrals each year from a baseline of $<1 \%$. We audited the service to measure impact and inform ongoing service development.

Methods We performed a retrospective audit of system 1 data and data prospectively collected by the RSC CNS for patients on the RSC caseload between April 2016 to August 2019. Data included referral source, reason, duration, outcome and information regarding intervention, advance care planning (ACP) and place of death.

Results 139 referrals were received for 110 patients. Mean duration of referral was 105 days range (1-1103). Referrals were made for symptom control (42\%), ACP (39\%), Psychosocial support (13\%), decision making support - dialysis versus conservative care $(6 \%)$ and stopping dialysis (2\%), patients imminently dying were referred to the non-disease specific CNS team. $49 \%$ of patients accessed another hospice service eg Day Hospice in addition to RSC CNS input. Faceto-face patient contacts occurred in patient's homes (83\%), outpatients (16\%) and hospital (1\%). 66 patients were discharged following a completed care episode, 29 of these were re-referred. 52 patients died, 38 (73\%) had their preferred place of death (PPD) recorded prior to death. In this group $1(3 \%)$ died in hospital. The rate of hospital death increased to $46 \%$ (6 out of 14 ) in patients without a documented PPD.

Conclusions The RSC service at StBH has increased access to specialist palliative care for people with advanced renal disease. Participation in ACP for these patients increased the likelihood of achieving a death outside of hospital.

\section{Survivorship and rehabilitation | poster 180}

\section{COST-EFFECTIVENESS OF COPD MANAGEMENT IN THE UK}

Mitri A Ng. King's College London

10.1136/spcare-2020-PCC.200

Introduction Chronic Obstructive Pulmonary Disease (COPD) is a long-term condition characterised by breathlessness. The
WHO has projected a $30 \%$ increase in total COPD deaths worldwide over the next decade. In 2012, COPD accounted for 29,776 deaths in the UK, and it costs the NHS over $£ 8,000,000$ annually.

Method This systematic review was conducted via searches on PubMed, using key words 'COPD', 'Management', 'Cost-effectiveness', 'exercise', 'QALY', 'UK' and 'Pulmonary Rehabilitation'. Studies with non-cost-effective interventions were removed. Studies using 'Quality Adjusted Life Years' and/or 'Incremental cost-effectiveness ratio' were considered for this review. Prices were adjusted for inflation.

Results Studies reviewed suggest that current standard care is cost-effective by NICE standards ( $£ 20,000$ to $£ 30,000$ per QALY). Cost effectiveness was improved with the addition of roflumilast to ICS/LABA therapy, where adding roflumilast led to an ICER of $£ 24,976$ per QALY gained for severe and very severe COPD in 2018. Tiotropium (£1934-£2548 in 2018] per QALY) proved to be a superior treatment over ipratropium ( $£ 2256-£ 2973$ in 2018] per QALY) and salmeterol ( $£ 2143-£ 2823$ in 2018] per QALY). Umeclidinium bromide added onto ICS/LABA therapy led to an ICER of $£ 1310$ per QALY, an improvement over Tiotropium. Adding pulmonary rehabilitation to treatment for COPD was likely to result in financial benefits, with mean incremental cost of adding PR was $-£ 152$ (- $£ 200$ in 2018$)$. Physical activity improved costeffectiveness versus sedentary lifestyle, reducing exacerbations and increasing QALYs.

Conclusion Current medical management is well optimized with regards to price per QALY gained. Umeclidinium should be considered for treatment instead of tiotropium. Physical Activity reduces exacerbations, increases QALYs and improves cost-effectiveness. However, data on cost-effectiveness of PR for COPD were insufficient. Furthermore, cost-effectiveness data of medical treatment should be updated as drug prices may have changed.

\section{Symptom management | posters $181-202$}

\section{IMPLEMENTING PATIENT CENTRED GOALS INTO A BREATHLESSNESS SYMPTOM MANAGEMENT CLINIC}

Camilla Barrow, Ruth Roberts. Birmingham St Mary's Hospice

\subsection{6/spcare-2020-PCC.201}

Introduction Space to breathe is a 5 week programme, with a rehabilitative focus, launched in January 2019 and designed through a Breathlessness steering group. A literature review recommended the use of the Goal Attainment Score (GAS) (Brighton 2018) and this was included into holistic measures of the breathlessness service. GAS light was chosen to reduce user burden and form part of the assessment of the service, complementing the rest of the Outcome Assessment Complexity Collaborative (OACC) suite and Chronic respiratory Questionnaire (CRQ) and Medical Research Council score of breathlessness (MRC) scores improved.

Method The programme is based on the Cambridge Breathing Thinking Functioning approach (Cambridge University Hospitals NHS Foundation Trust 2019) and with a focus on mastery of symptoms of breathlessness. Sessions include taught educational sessions followed by group discussions and exercise. Participants were supported by carers and clinicians to set patient identified SMART goals. GAS light 